\title{
Simultaneous Piezoelectric Actuator and Sensor Placement Optimization and Control Design of Manipulators with Flexible Links Using SDRE Method
}

\author{
Alexandre Molter, ${ }^{1}$ Otávio A. Alves da Silveira, ${ }^{2}$ \\ Jun S. Ono Fonseca, ${ }^{2}$ and Valdecir Bottega ${ }^{1}$ \\ ${ }^{1}$ Department of Mathematics and Statistics, Federal University of Pelotas, s/n ${ }^{\circ}$, 354, 96010-900 Pelotas, \\ RS, Brazil \\ ${ }^{2}$ Department of Mechanical Engineering, Federal University of Rio Grande do Sul, R. Sarmento Leite, 425, \\ 90050-170 Porto Alegre, RS, Brazil
}

Correspondence should be addressed to Valdecir Bottega, valdecir.bottega@ufpel.edu.br

Received 17 June 2010; Accepted 22 November 2010

Academic Editor: Sergio Preidikman

Copyright (c) 2010 Alexandre Molter et al. This is an open access article distributed under the Creative Commons Attribution License, which permits unrestricted use, distribution, and reproduction in any medium, provided the original work is properly cited.

\begin{abstract}
This paper presents a control design for flexible manipulators using piezoelectric actuators bonded on nonprismatic links. The dynamic model of the manipulator is obtained in a closed form through the Lagrange equations. Each link is discretized using finite element modal formulation based on Euler-Bernoulli beam theory. The control uses the motor torques and piezoelectric actuators for controlling vibrations. An optimization problem with genetic algorithm (GA) is formulated for the location and size of the piezoelectric actuator and sensor on the links. The natural frequencies and mode shapes are computed by the finite element method, and the irregular beam geometry is approximated by piecewise prismatic elements. The State-Dependent Riccati Equation (SDRE) technique is used to derive a suboptimal controller for a robot control problem. A state-dependent equation is solved at each new point obtained for the variables from the problem, along the trajectory to obtain a nonlinear feedback controller. Numerical tests verify the efficiency of the proposed optimization and control design.
\end{abstract}

\section{Introduction}

The development of lightweight structures has attracted research attention on coping with flexibility effects. Structures with a reduced weight are essential to improve the performance in mobile applications, such as flexible robots, aircrafts, and spacecrafts. The design of these structures requires a control system, which takes into account the interaction of the applied forces and the elastic modes. Structure vibration suppression depends not only on control 
design, but also on the sensor/actuator (S/A) selection and placement [1]. This paper considers piezoelectric $\mathrm{S} / \mathrm{A}$ pairs bounded on a beam.

Piezoelectric coupling is defined as a relation between an applied electric field and mechanical strain, or an applied strain and electric field in certain crystals, ceramics, and films. In general, flexible robot manipulators feature surface bonded or embedded piezoelectric actuators and/or sensor. The piezoelectric actuator generates a large actuating force and has a fast response time. Moreover it is smaller than other actuating systems as electrical motor or hydraulics for the same force [2].

A flexible beam optimization and control design is composed by two parts: control gain and the placement of actuators and sensors. The proposed control must stabilize the system against the motion-induced vibration. Design of a smart structure system requires more than accurate structural modeling, since both structural dynamics and control need to be considered for active vibration control [3]. A formulation of the dynamic equations, in modal space, can be seen in Abreu et al. [4]. Optimal control design for location and size and feedback gain is presented in some works $[1,3,5]$. This paper presents a genetic algorithm (GA) design for S/A placement and size, considering maximal system energy dissipation $[6,7]$. A limited number of S/A pairs was considered distributed on the beam. Sun et al. [2] suggest more than one actuator and consider linear velocity feedback.

Flexible structures can be built in complex geometries, which cannot be modeled by simple beam bending equations. In this paper, the finite element method (FEM) is used for dealing complex geometry within the realm of the Euler-Bernoulli beam theory. This paper proposes a piezoelectric control with velocity feedback gain. The piezoelectric actuators and sensors are fixed without considering the adhesive layer influence.

In flexible structure, a piezoelectric actuator is applied to single-link flexible manipulators in [8-10] and applied to two-link flexible manipulators in [11]. These works considered control torque of the motor, determined based on the rigid link dynamics and the oscillations caused by the torque are suppressed by applying a feedback control voltage to the piezoelectric actuator.

Robotic systems are modeled as linear with respect to parameters as mass, inertia, and damping factors, but this assumption is not valid for the state, requiring nonlinear control design. The SDRE [12] is among the techniques that emerged to deal with highly non-linear and complex systems, such as the control of flexible robotic dynamics. The SDRE nonlinear regulator produces a closed-loop solution which is locally asymptotically stable [13, 14]. The procedure to drive the tip position to a desired point via SDRE technique can consider successive optimal solutions for static equations and feedback control stabilized system.

In this paper we propose a minimum energy positioning control technique for a robot arm with flexible links, where the motor torque controls the joint angle tracking and reduces the low frequency vibrations on the links. Piezoelectric sensors and actuators are added to control the high frequency vibrations beyond the torque control. The lower fundamental modes are responsible for the most of the beam tip displacement, therefore the first two eigenfunctions are considered in the paper. Simulation code was created to assess the control model feasibility and efficiency.

The remainder of this paper is organized as follows: the mathematical formulation of the dynamic model of flexible links with piezoelectric material and a numerical approximation for the vibration modes is presented in the next section. Piezoelectric material control design is discussed in Section 3; an energy approach for location and size of S/A pairs is presented in Section 4 and the genetic algorithm design in Section 5; physical parameters of the model and partial results for location and size of S/A pairs are discussed in Section 6; 


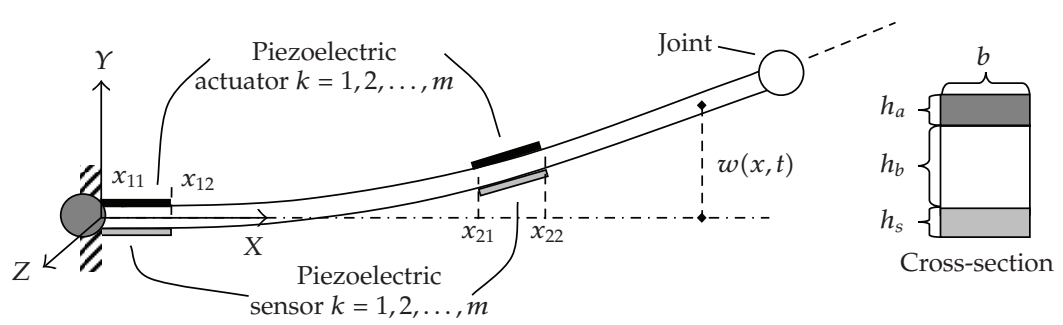

Figure 1: A flexible structure with piezoelectric actuators and sensors.

the uncoupled control designs via bounded piezoelectric material and via SDRE methodology are summarized in Section 7; numerical results are discussed in Section 8; conclusions and final considerations follow in Section 9.

\section{Dynamic Model of Flexible Links with Piezoelectric Material Pairs}

Consider a uniform robot link featuring some piezoelectric actuators bonded on the top face and sensors bonded on the bottom face, as shown in Figure 1.

This structure can be modeled as an Euler-Bernoulli beam. Assuming $m \mathrm{~S} / \mathrm{A}$ pairs, when external charges are applied on the actuators, one can write, from the Hamilton principle, the following partial differential equation [15]:

$$
\frac{\partial^{2}}{\partial x^{2}}\left(E I \frac{\partial^{2} w(x, t)}{\partial x^{2}}\right)+\rho A \frac{\partial^{2} w(x, t)}{\partial t^{2}}=\sum_{k=1}^{m} \frac{\partial^{2} M_{k}^{a}(x, t)}{\partial x^{2}}
$$

where $w$ is the deflection of neutral axis, $E, I, \rho, b$, and $A$ are Young's modulus, inertia moment, density, width, and cross-sectional area of the model, respectively, and $M_{k}^{a}$ is the force moment induced by actuator $k$, given by [4]

$$
M_{k}^{a}(x, t)=\int_{h_{b} / 2}^{h_{b} / 2+h_{a}} \sigma_{x} b y d y=\int_{h_{b} / 2}^{h_{b} / 2+h_{a}} \frac{d_{31 a} E_{a} V_{k}^{a}(x, t)}{h_{a}} b y d y,
$$

where $h_{a}, h_{s}$ sand $h_{b}$ are the actuator, sensor, and beam thicknesses, respectively; $d_{31 a}$ and $E_{a}$ are the piezoelectric strain constant and Young's modulus of the actuator; $V_{k}^{a}(x, t)$ is the voltage applied to actuator $k$. The evolution of the integral (2.2) results the following expression

$$
M_{k}^{a}=b d_{31 a} E_{a} r_{a} V_{k}^{a}(x, t),
$$

where $r_{a}$ denotes the distance measured from the neutral surface of the beam to the midplane of the actuator. The voltage distribution of actuator $k$ can be expressed as $V_{k}^{a}(x, t)=$ $V_{k}^{a}(t)\left[H_{a}\left(x-x_{k 1}\right)-H_{a}\left(x-x_{k 2}\right)\right]$, where $H_{a}$ is the Heaviside functions for generalized location and $x_{k 1}$ and $x_{k 2}$ are the end coordinates of the actuator $k$. Details of derivation of equation (2.1) can be seen in Abreu et al. [4]. 
Assuming a uniform voltage on the electrode surface of actuator $k$, the moment can be expressed as

$$
M_{k}^{a}=P_{k}^{a} V_{k}^{a}(t),
$$

where $P_{k}^{a}=b d_{31 a} E_{a} r_{a}$.

\subsection{Finite Element Formulation}

In order to find the mode shapes and natural frequencies, as well as to solve the control problem, one can consider an Euler-Bernoulli finite element model with a total of dof-degrees of freedom, with $m$ piezoelectric S/A pairs. The two-node element presents two degrees of freedom in each node, deflection, and slope. The inclusion of the piezoelectric material on the flexible model is accounted by defining the beam properties elementwise, but the bonding is neglected. In the elements where the material is added, the geometry and stiffness are changed. In this manner, where the standard two-node cubic Hermite interpolation is used and considering damping, (2.1) can be rewritten as

$$
\mathbf{M u}+\mathbf{D} \dot{\mathbf{u}}+\mathbf{K u}=\mathbf{P}^{\mathbf{a}} \mathbf{v}^{\mathbf{a}},
$$

where $\mathbf{M}, \mathbf{D}$, and $\mathbf{K}$ are the global $($ dof $\times$ dof $)$ mass, damping, and stiffness matrices. The applied voltage $\mathbf{v}^{\mathbf{a}}$ is assumed to be uniform over the element and is an $m$-dimensional vector. The energetic equivalent generalized force $\mathbf{P}^{\mathbf{a}}$ is a $($ dof $\times m)$ matrix which maps the applied voltage to the induced displacements and can be computed by the element contributions

$$
\mathbf{P}_{\text {elem }}^{\mathbf{a}}=b d_{31 a} E_{a}\left[\begin{array}{llll}
0 & r_{a} & 0 & -r_{a}
\end{array}\right]^{T} \text {. }
$$

Here, one can note that $\mathbf{P}^{\mathbf{a}}$ actuates on the slopes, that is, as a moment. Finally the nodal displacements $\mathbf{u}$, neglecting axial deflection, are given by

$$
\mathbf{u}=\left[\begin{array}{lllllll}
w_{1} & \theta_{1} & w_{2} & \theta_{2} & \cdots & w_{\text {node }} & \theta_{\text {node }}
\end{array}\right]^{T},
$$

where $w$ are the nodal transverse deflections and $\theta=\partial w / \partial x$ are the slopes.

The beam was considered flexible and nonprismatic, therefore subject to motion induced vibration, which affects the trajectory of the endpoint. Equations (2.8) and (2.9) show a possibility to consider nonprismatic beams, using a specifically formulation for computation. An elementwise prismatic approach is adopted for the nonprismatic beam, where the mass matrix of each element is computed considering an average sectional area given by

$$
A_{\text {elem }}=\frac{A_{1}+\sqrt{A_{1}+A_{2}}+A_{2}}{3}
$$


and the stiffness matrix uses an average bending stiffness given by

$$
E I_{\text {elem }}=\frac{E I_{1}+\sqrt[4]{\left(E I_{1}\right)^{3} E I_{2}}+\sqrt{E I_{1} E I_{2}}+\sqrt[4]{E I_{1}\left(E I_{2}\right)^{3}}+E I_{2}}{5},
$$

where the index 1 refers to the first node of the element and the index 2 to the second.

For the elements with piezoelectric material [16], the flexural stiffness is considered as

$$
\begin{aligned}
E I= & E_{a}\left[\frac{h_{a}^{3} b}{12}+h_{a} b\left(h_{s}+\frac{h_{a}}{2}+h_{b}-d_{n}\right)^{2}\right]+E_{b}\left[\frac{h_{b}^{3} b}{12}+h_{b} b\left(h_{s}+\frac{h_{b}}{2}-d_{n}\right)^{2}\right] \\
& +E_{S}\left[\frac{h_{s}^{3} b}{12}+h_{s} b\left(h_{s}-d_{n}\right)^{2}\right],
\end{aligned}
$$

where the subscripts $a, b$, and $s$ refer to actuator, beam, and sensor respectively; $d_{n}$ is the distance from the bottom of the sensor to the neutral axis.

\subsection{Modal Analysis}

The finite element modal problem is solved by a classical eigenproblem solution method. However, one can consider the time and space separability principle to the control analysis. Thus, the displacement can be written as

$$
\mathbf{u}=\sum_{i=1}^{\infty} \phi_{i}(x) \eta_{i}(t) \approx \sum_{i=1}^{n} \phi_{i}(x) \eta_{i}(t)=\mathbf{\Phi} \eta
$$

where $\phi_{i}(x)$ are the mass normalized orthogonal mode shapes and $\eta_{i}(t)$ are generalized modal amplitudes. Truncating the representation of $\mathbf{u}$ to $n$ modes, the dynamics of the flexible structure with $m$ distributed piezoelectric S/A pairs, in terms of modal coordinates, can be expressed as

$$
\mathrm{M \Phi} \ddot{\eta}+\mathrm{D} \Phi \dot{\eta}+\mathrm{K} \Phi \eta=\mathbf{P}^{\mathrm{a}} \mathbf{v}^{\mathrm{a}}
$$

where $\boldsymbol{\Phi}$ is the truncated modal matrix and $\eta=\left[\eta_{1} \eta_{2} \cdots \eta_{n}\right]^{\mathrm{T}}$ is a vector of modal amplitudes.

Premultiplying both sides by $\boldsymbol{\Phi}^{\mathrm{T}},(2.12)$ can be transformed in the reduced modal space as

$$
\overline{\mathbf{M}} \ddot{\eta}+\overline{\mathbf{D}} \dot{\eta}+\overline{\mathbf{K}} \eta=\overline{\mathbf{P}}^{\mathrm{a}} \mathbf{v}^{\mathrm{a}}
$$

where

$$
\overline{\mathbf{M}}=\Phi \mathbf{\Phi}, \quad \overline{\mathbf{D}}=\Phi D \Phi, \quad \overline{\mathbf{K}}=\Phi \text { ФФ, } \quad \overline{\mathbf{P}}^{\mathrm{a}}=\Phi^{\mathrm{T}} \mathbf{P}^{\mathrm{a}}
$$


are $(n \times n)$ diagonal matrices because of the orthogonality of the eigenvectors, except $\overline{\mathbf{P}}^{\mathbf{a}}$ which is $(n \times m)$ matrix and represents the actuator force.

In open state space form (2.13) can be rewritten as

$$
\begin{gathered}
\dot{\boldsymbol{\xi}}=\mathbf{A} \boldsymbol{\xi}+\mathbf{B v}^{\mathbf{a}}, \\
\mathbf{A}=\left[\begin{array}{cc}
\mathbf{0}_{n \times n} & \mathbf{I}_{n \times n} \\
-\overline{\mathbf{M}}^{-1} \overline{\mathbf{K}} & -\overline{\mathbf{M}}^{-1} \overline{\mathbf{D}}
\end{array}\right], \quad \mathbf{B}=\left[\begin{array}{c}
\mathbf{0}_{n \times m} \\
-\overline{\mathbf{M}}^{-1} \overline{\mathbf{P}}^{\mathbf{a}}
\end{array}\right],
\end{gathered}
$$

where $\boldsymbol{\xi}=\left[\begin{array}{ll}\eta & \dot{\eta}\end{array}\right]^{\mathrm{T}}$ is the state vector, A is the system matrix $(2 n \times 2 n)$, B is the control matrix $(2 n \times m)$, and $\mathbf{v}^{\mathrm{a}}$ is the control input vector of the actuator.

\subsection{Sensor Equations}

The sensors are considered with the same length and axial position than the actuators, but vertically opposite (Figure 1). On a sensor $k$, the open circuit voltage $V_{k}^{s}(t)$ due to the bending effect can be estimated by the normal strains in the axial direction $\varepsilon_{x}$ of the beam. For each sensor, for a specific vibration mode, it is given by

$$
\begin{aligned}
V_{k}^{s}(t) & =\frac{h_{s}}{S_{e}} \int_{S_{e}}^{S_{e}} h_{31 s} \varepsilon_{x} d S_{e}=-\frac{h_{s}}{x_{k 2}-x_{k 1}} \int_{x_{1(k)}}^{x_{2(k)}} h_{31 s} \varepsilon_{x} d x \\
& =-\frac{h_{s}}{x_{k 2}-x_{k 1}} \int_{x_{1(k)}}^{x_{2(k)}} g_{31 s} E_{s} r_{s} \frac{\partial^{2} w}{\partial x^{2}} d x=C_{k}^{s}\left[\theta\left(x_{2}\right)-\theta\left(x_{1}\right)\right],
\end{aligned}
$$

where $h_{s}$ is the sensor thickness, $S_{e}$ is the electrode surface, and $h_{31 s}$ is the piezoelectric constant, $C_{k}^{s}=-\left[h_{s} /\left(x_{k 2}-x_{k 1}\right)\right] g_{31 s} E_{s} r_{s} ; E_{s}$ is the Young's modulus of the sensor, $r_{s}$ is the distance measured from the neutral axis of the beam to the midplane of the sensor layer, and $g_{31 s}$ is the piezoelectric stress constant [17]. The input voltage applied to the conjugated actuator is determined by using the control law discussed below after obtaining the sensor output voltage.

\section{Piezoelectric Material Control Design}

In this paper it is proposed a constant gain negative velocity feedback control to the actuator [16], expressed as

$$
V^{a}(t)=-G \dot{V}^{s}(t)
$$

where $G$ is the feedback gain. $V^{s}(t)$ is the voltage generated by the sensor, obtained by integrating the electric charge developed on the piezoelectric sensor surface, (2.16). 
Returning to the right side of (2.12), one can write

$$
\begin{aligned}
P^{\mathrm{a}} \mathbf{v}^{\mathrm{a}} & =-\mathrm{P}^{\mathrm{a}} \mathrm{G} \dot{\mathbf{v}}^{\mathrm{s}} \\
& =-\mathrm{P}^{\mathrm{a}} \mathrm{G} C^{\mathrm{s}} \dot{\mathbf{u}} \\
& \approx-\mathrm{P}^{\mathrm{a}} \mathrm{G} C^{\mathrm{s}} \boldsymbol{\Phi} \dot{\eta}
\end{aligned}
$$

where $\mathbf{G}$ is an $(m \times m)$ feedback gain matrix and $\mathbf{C}^{\mathbf{s}}$ is an $(m \times d o f)$ output sensor matrix. The resulting $\mathrm{S} / \mathrm{As}$ control law for the system equation is expressed as

$$
\overline{\mathbf{M}} \ddot{\eta}+\overline{\mathbf{D}} \dot{\eta}+\overline{\mathbf{K}} \eta=-\overline{\mathbf{P}}^{\mathrm{a}} \mathrm{G} \overline{\mathrm{C}}^{\mathrm{s}} \dot{\eta} .
$$

Using the sensor equations and the proposed control, (2.15) can be expressed in the corresponding closed-loop state space form as

$$
\begin{gathered}
\dot{\xi}=(\mathbf{A}+\mathbf{B}) \boldsymbol{\xi}=\mathbf{A}_{\mathrm{cl}} \xi, \\
\mathbf{A}_{\mathrm{cl}}=\left[\begin{array}{cc}
\mathbf{0}_{n \times n} & \mathbf{I}_{n \times n} \\
-\overline{\mathbf{M}}^{-1} \overline{\mathbf{K}} & -\overline{\mathbf{M}}^{-1} \overline{\mathbf{D}}-\overline{\mathbf{M}}^{-1} \overline{\mathbf{P}}^{\mathrm{a}} \mathbf{G} \overline{\mathbf{C}}^{\mathrm{s}}
\end{array}\right] .
\end{gathered}
$$

Since the mode shapes are mass normalized, the following simplifications are valid:

$$
\begin{gathered}
\overline{\mathbf{M}}=\mathbf{I}=\operatorname{diag}\left(\delta_{i i}\right), \\
\overline{\mathbf{M}}^{-1} \overline{\mathbf{K}}=\boldsymbol{\Omega}^{2}=\operatorname{diag}\left(\omega_{i}^{2}\right), \\
\overline{\mathbf{M}}^{-1} \overline{\mathbf{D}}=2 \zeta \boldsymbol{\Omega}=\operatorname{diag}\left(2 \zeta_{i} \omega_{i}\right),
\end{gathered}
$$

where $\delta_{i i}$ is the Kronecker delta, $\omega_{i}$ and $\zeta_{i}$ are the natural frequency and structural damping ratio of $i$ th vibration mode. The actuator force, feedback gain, and output matrices can be computed as

$$
\begin{gathered}
\overline{\mathbf{P}}^{\mathbf{a}} \mid \bar{P}_{i k}^{a}=E_{a} d_{31 a} b r_{a}\left[\theta_{i}\left(x_{k 2}\right)-\theta_{i}\left(x_{k 1}\right)\right], \\
\mathbf{G}=\operatorname{diag}\left(G_{k}\right), \\
\overline{\mathbf{C}}^{\mathrm{s}} \mid \overline{\mathrm{C}}_{k i}^{s}=-\frac{h_{s}}{x_{k 2}-x_{k 1}} E_{s} g_{31 s} r_{s}\left[\theta_{i}\left(x_{k 2}\right)-\theta_{i}\left(x_{k 1}\right)\right] .
\end{gathered}
$$

\section{Energy Approach for Location and Size of S/A Pairs Optimal Design}

Controlling structural vibration depends not only on the control law, but also on the selection and location of the actuators and sensors [18]. This paper proposes feedback control for the actuator and sensor placement and size optimization, based on maximizing the control energy dissipation [1]. This procedure takes into account the mass and stiffness of actuators 
and sensors and their effect on the mechanical behavior of the structure. This influence is combined to the control characteristics to obtain an objective function that depends on the actuators location and size and the feedback gain.

The dynamic of the beam with $m$ piezoelectric sensors and actuators is expressed in (3.3), in terms of modal coordinates.

Theorem 4.1. The total energy stored in the system can be considered a Lyapunov function as

$$
W=T+U=\frac{1}{2} \dot{\eta}^{\mathrm{T}} \overline{\mathbf{M}} \dot{\eta}+\frac{1}{2} \boldsymbol{\eta}^{\mathrm{T}} \overline{\mathbf{K}} \boldsymbol{\eta}>0
$$

where $T$ is the kinetic energy, $U$ is the potential energy, and $\boldsymbol{\eta}$ is the generalized coordinates vector, associated with beam deflections.

Proof. The proof of this theorem presents no great difficulties. We needed to show that the derivative of the function (4.1) is negative definite. Differentiating it with respect to time gives

$$
\dot{W}=\dot{T}+\dot{U}=\frac{1}{2} \dot{\eta}^{\mathrm{T}} \dot{\overline{\mathbf{M}}} \dot{\eta}+\dot{\eta}^{\mathrm{T}} \overline{\mathbf{M}} \ddot{\eta}+\frac{1}{2} \eta^{\mathrm{T}} \dot{\overline{\mathbf{K}}} \boldsymbol{\eta}+\dot{\boldsymbol{\eta}}^{\mathrm{T}} \overline{\mathbf{K}} \eta
$$

The terms $\dot{\eta}^{\mathrm{T}} \dot{\overline{\mathbf{M}}} \dot{\eta}$ and $\eta^{\mathrm{T}} \dot{\overline{\mathbf{K}}} \eta$ are equal to zero, since the matrices $\overline{\mathbf{M}}$ and $\overline{\mathbf{K}}$ are time independent for the beam. Isolating $\dot{\eta}^{\mathrm{T}}$ and using (3.3) yield

$$
\dot{W}=\dot{T}+\dot{U}=-\dot{\eta}^{\mathrm{T}} \overline{\mathrm{D}} \dot{\boldsymbol{\eta}}-\dot{\boldsymbol{\eta}}^{\mathrm{T}} \overline{\mathbf{P}}^{\mathrm{a}} \mathrm{G} \overline{\mathrm{C}}^{\mathrm{s}} \dot{\boldsymbol{\eta}}<0,
$$

where the first and the second terms describe the removed system energy rates by the internal damping and by the control feedback, respectively. In this manner $\dot{W}$ is negative definite.

Integrating (4.3) results

$$
W\left(t_{0}\right)=W_{f}+W_{c}=\int_{t_{0}}^{\infty} \dot{\boldsymbol{\eta}}^{\mathrm{T}} \overline{\mathbf{D}} \dot{\boldsymbol{\eta}} d t+\int_{t_{0}}^{\infty} \dot{\boldsymbol{\eta}}^{\mathrm{T}} \overline{\mathbf{P}}^{\mathrm{a}} \mathbf{G} \overline{\mathbf{C}}^{\mathrm{s}} \dot{\boldsymbol{\eta}} d t
$$

where $W\left(t_{0}\right)$ denote the initial energy of the system, $W_{f}$ and $W_{c}$ represent energy dissipated by internal damping and by the control action, respectively.

The quadratic cost function for the regulator problem is considered for maximizing the energy dissipation

$$
W_{c}=\int_{0}^{\infty} \xi^{\mathrm{T}} \mathbf{Q} \xi d t
$$


where $\mathbf{Q}$ is positive semidefinite weighting matrix, and their elements are selected connecting output of the sensor feedback with the input on the actuator

$$
\mathbf{Q}=\left[\begin{array}{cc}
\mathbf{0}_{n \times n} & \mathbf{0}_{n \times n} \\
\mathbf{0}_{n \times n} & \overline{\mathbf{P}}^{\mathrm{a}} \mathrm{G} \overline{\mathbf{C}}^{\mathrm{s}}
\end{array}\right] .
$$

Equation (4.5) can be reduced, by standard-state transformation techniques, to the expression

$$
W_{c}=\boldsymbol{\xi}_{0}^{\mathrm{T}} \mathbf{P} \xi_{0}
$$

where $\boldsymbol{\xi}_{0}$ are the initial conditions. The determination of the matrix $\mathbf{P}$ can be reduced to solving a matrix Lyapunov equation

$$
\mathbf{A}_{\mathrm{cl}}^{\mathrm{T}} \mathbf{P}+\mathbf{P A}_{\mathrm{cl}}+\mathbf{Q}=\mathbf{0}
$$

For effective vibration suppression, it is reasonable to derive a method to increase the energy dissipated by the control. It is well known that $W_{c}$ depends on the placement and the size of the piezoelectric pairs, as well as the feedback matrix gain. Therefore, $W_{c}$ can be used as an optimization criterion to determine locations and size [1]. Since, one wishes to maximize the energy dissipated, one can write

$$
\operatorname{minimize} J(x, L)=-W_{c} \text {. }
$$

It is noticeable that $W_{c}$ depends on the initial conditions of the flexible structure. In some works, such as $[1,6,19]$, in order to eliminate this dependence, it is assumed an initial state as a random variable. The initial condition is modeled as a random variable uniformly distributed on the surface of the $2 n$-dimensional unit sphere, then the expected value of $J^{*}$ scaled by $n$ can be given by [20].

$$
J^{*}=\int_{0}^{T} \operatorname{tr}\left(\xi^{\mathrm{T}} \overline{\mathbf{P}}^{\mathrm{a}} \mathrm{GC}^{\mathrm{S}} \boldsymbol{\xi}\right) d t=\operatorname{tr}(\mathbf{P})
$$

where $\operatorname{tr}(\mathbf{P})$ represent the trace of matrix $\mathbf{P}$. The stability of the feedback matrix $\mathbf{A}_{\mathbf{c l}}$ is an important condition for the existence of the feedback control. It can be shown that for our problem the stability for $\mathbf{A}_{\mathbf{c l}}$ is assured.

\section{Genetic Algorithm}

In this section, a genetic algorithm optimization problem is formulated to find the placement $\left(x_{k}\right)$ and sizes $\left(L_{k}\right)$ of the $m$ piezoelectric S/A pairs bonded onto the beam, which minimize $J$ given by (4.10). Genetic algorithms basically are random adaptive search techniques derived from the Darwinian evolutionary principle of "survival of the fittest." The design variables are coded as a string that corresponds to the individual chromosome, and the objective 
function value corresponds to the fitness. The artificial recombination among the population of strings (individuals) is based on the fitness and the accumulated knowledge. A new set of individuals is created by using parent selection, crossover, and mutation from the old set of individuals in every new generation.

Each individual is coded in a form that the first chromosome contains $m$ binary positions with $n 1$ genes each one, that is, each S/A pair can take $2^{n 1}$ possible positions in the beam. The second chromosome shows $m$ integer numbers to encode the size, of a group with $n 2$ possible values. Thus, the binary-integer encoded GA is developed and implemented.

The parent selection is done using a tournament operator based in [21]. Since the initial population is random and using the crossover and mutation operators, the S/A pairs can be out of order or overlapped (infeasible solutions). Therefore, a penalization was considered to measure the constraint violation, given by

$$
g= \begin{cases}\sum_{k=2}^{m}\left(x_{k 1}-x_{(k-1) 2}\right) & \text { if } x_{k 1}<x_{(k-1) 2} \\ 0, & \text { otherwise. }\end{cases}
$$

In the tournament, two individuals are compared at a time, and the following criteria are always enforced.

(i) Any feasible solution is preferred to any infeasible solution.

(ii) Among two feasible solutions, the one having better objective function value is preferred.

(iii) Among two infeasible solutions, the one having smaller constraint violation is preferred.

The binary-encoded part uses one-point crossover, and the mutation is done in one gene. For the integer-encoded part, the crossover is similar with the standard binary-encoded GA; the only difference is that the base of the integer-encoded GA is $n 2$. Elitism is used to increase the performance, and the stop criterion is the total number of generations.

\section{Simulations for S/A Pairs Optimization}

This section presents the results for a cantilever beam (analogically for robot links) to verify the feasibility and reliability of the optimization technique presented before. In the first example, the beam, actuators, and sensors present a similar stiffness and mass density. In the second one, the sensor is a piezofilm, so it is more flexible than the piezoceramic used in the first one. Moreover, the aluminum beam is lighter than the steel beam.

Example 6.1. In this example the nonzero feedback matrix gain values $\mathrm{G}$ are set as a constant 1 (one). Structural damping ratio for any vibration mode is equal to 0.1 . Table 1 presents mechanical and geometrical properties of the beam and piezoelectric actuators and sensors. The initial conditions of the generalized coordinate vector, in this case, are given by

$$
\begin{aligned}
\boldsymbol{\eta}(0)^{T} & =\left[\begin{array}{lll}
0.1 & 0.1 & 0.1
\end{array}\right] \\
\dot{\boldsymbol{\eta}}(0)^{T} & =\left[\begin{array}{lll}
0.1 & 0.1 & 0.1
\end{array}\right]
\end{aligned}
$$


Table 1: Beam and piezoelectric properties (Example 6.1).

\begin{tabular}{lccc}
\hline & Beam & Actuators & Sensors \\
\hline Young's modulus $(\mathrm{GPa})$ & $210 \times 10^{9}$ & $139 \times 10^{9}$ & $139 \times 10^{9}$ \\
Mass density $\left(\mathrm{kg} \mathrm{m}^{-3}\right)$ & 7500 & 7810 & 7810 \\
Stress constant $d_{31}\left(\mathrm{~m} \mathrm{~V}^{-1}\right)$ & - & $11 \times 10^{-11}$ & - \\
Strain constant $g_{31}\left(\mathrm{Vm} \mathrm{N}^{-1}\right)$ & - & - & 0.01 \\
Thickness $(\mathrm{m})$ & $2 \times 10^{-3} \rightarrow 1.6 \times 10^{-3}$ & $40 \times 10^{-6}$ & $40 \times 10^{-6}$ \\
Width $(\mathrm{m})$ & 0.03 & 0.03 & 0.03 \\
Length $(\mathrm{m})$ & 0.5 & - & - \\
\hline
\end{tabular}

Table 2: Optimal placement and size of S/A.

\begin{tabular}{|c|c|c|c|c|c|c|}
\hline \multirow{3}{*}{ Pairs } & \multicolumn{6}{|c|}{ Modes } \\
\hline & \multicolumn{2}{|c|}{$1 \mathrm{st}$} & \multicolumn{2}{|c|}{ 1st and 2nd } & \multicolumn{2}{|c|}{ 1st, 2nd and 3rd } \\
\hline & $x_{k}(\mathrm{~m})$ & $L_{k}(\mathrm{~m})$ & $x_{k}(\mathrm{~m})$ & $L_{k}(\mathrm{~m})$ & $x_{k}(\mathrm{~m})$ & $L_{k}(\mathrm{~m})$ \\
\hline 1 & 0.0000 & 0.200 & 0.0000 & 0.200 & 0.0000 & 0.075 \\
\hline 2 & 0.2063 & 0.100 & 0.2063 & 0.200 & 0.1269 & 0.125 \\
\hline 3 & 0.5000 & 0.050 & 0.5000 & 0.050 & 0.3333 & 0.100 \\
\hline
\end{tabular}

Using the genetic algorithm proposed in Section 5, the placement optimization of three S/A pairs in a cantilever beam with 64 possible positions is presented. There are four possible sizes for each pair, $L_{k}=\left[\begin{array}{llll}0.050 & 0.075 & 0.100 & 0.200\end{array}\right] \mathrm{mm}$. The population size and the number of generations are 300 and 100, respectively. The variation in the results for one to four vibration modes controlled simultaneously is investigated. Table 2 shows the optimal positions in relation to the number of combined controlled modes. The convergence of the fitness function for three modes combined is shown in Figure 2. Note that the fitness stabilized when the number of generation is around 15; after that, no chances of fitness appear.

The positions found for the third piezoelectric pair for one and two vibration modes combined $\left(x_{31}=0.5 \mathrm{~m}\right)$ mean that the last S/A pair is out of the beam. This position is the 64th possibility.

With this result, for location and size of the S/A pairs, it is noticeable that the control gain depends on the location, and this can help in the effectiveness of the vibration control.

Example 6.2. The results obtained in this example were used in the continuity of this paper to find the best locations and sizes for the S/A pairs in the flexibly link. Again, the non-zero feedback matrix gain values $\mathrm{G}$ are set as a constant 1 (one). Structural damping ratio is equal to 0.008 and 0.005 , for the first and second modes, respectively. Table 3 presents mechanical and geometrical properties of the beam and piezoelectric actuators and sensors. The initial conditions are the same of the previous example.

Figures 3 (case 1) and 4 (case 2) show the objective function that depends on $x_{k}$ and $L_{k}$ variables of a second S/A pair. The values results were obtained considering two vibration modes. In the first case, an S/A pair with length $0.1 \mathrm{~m}$ was fixed in the origin. It can be seen clearly in this figure, that the best place and length for the second pair are $0.10 \mathrm{~m}$ and $0.15 \mathrm{~m}$, respectively. For the second case, an S/A pair with length $0.15 \mathrm{~m}$ was fixed in the origin. And now, it can be noted that there are two possible solutions with almost the same value for the 


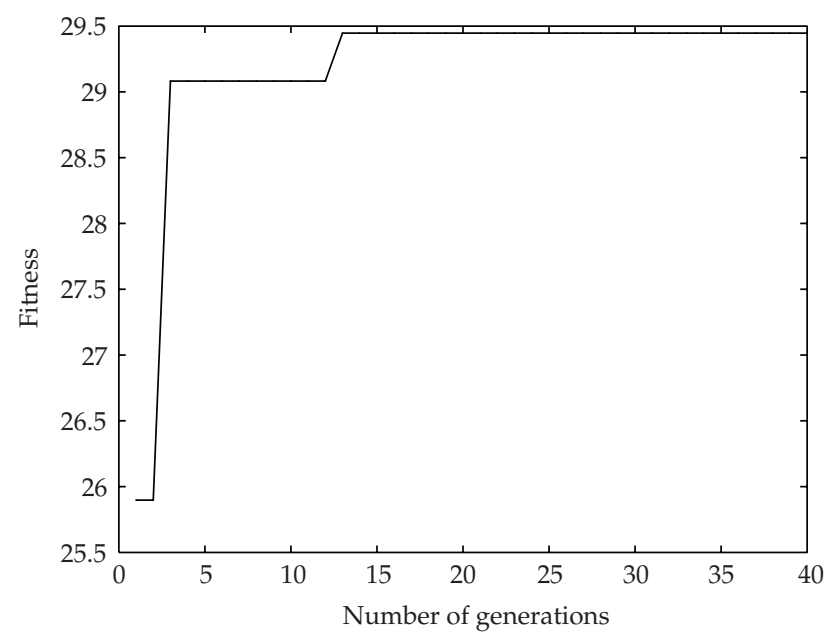

Figure 2: Convergence of the fitness function for 3 vibration modes.

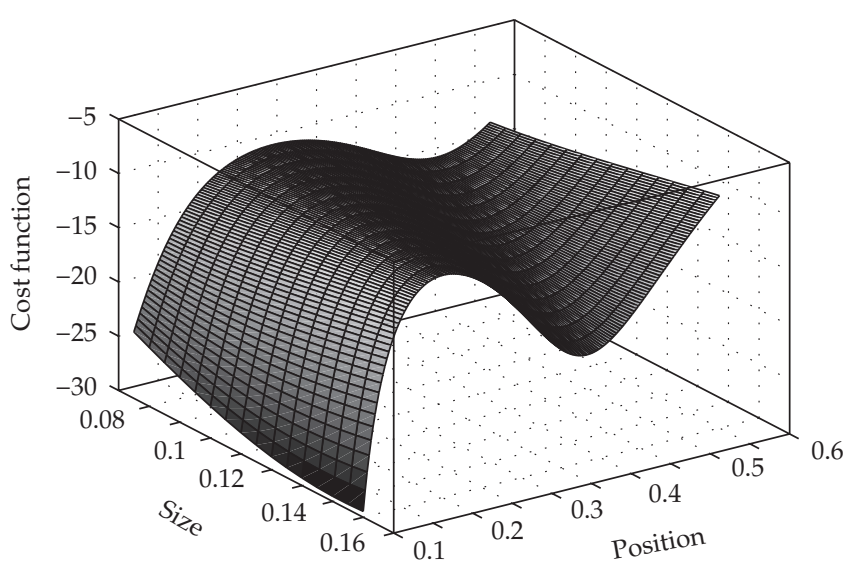

Figure 3: Dissipated energy objective function due to piezoelectric control (case 1).

objective function. The first possible position for the second pair is right after the first one at $0.15 \mathrm{~m}$ with size $0.10 \mathrm{~m}$. And the second possible solution is approximately at $0.35 \mathrm{~m}$ with size $0.15 \mathrm{~m}$.

Considering the characteristics of the first case (an S/A pair with length $0.1 \mathrm{~m}$ fixed in the origin) a new GA was performed. The objective of the problem is to find the placement and size of the second S/A pair in a cantilever beam with 64 possible positions and four

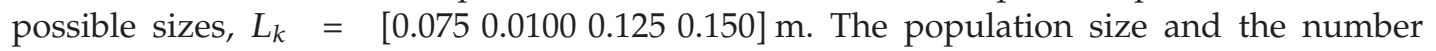
of generations are 300 and 100, respectively. The position and size found for the second piezoelectric was $0.1 \mathrm{~m}$ and $0.15 \mathrm{~m}$, as we expected from Figure 3. This result will be used in the continuity of this paper. The first two natural frequencies obtained for the flexible link (considered as a cantilever beam) are $3 \mathrm{~Hz}$ and $12.7 \mathrm{~Hz}$, respectively. 


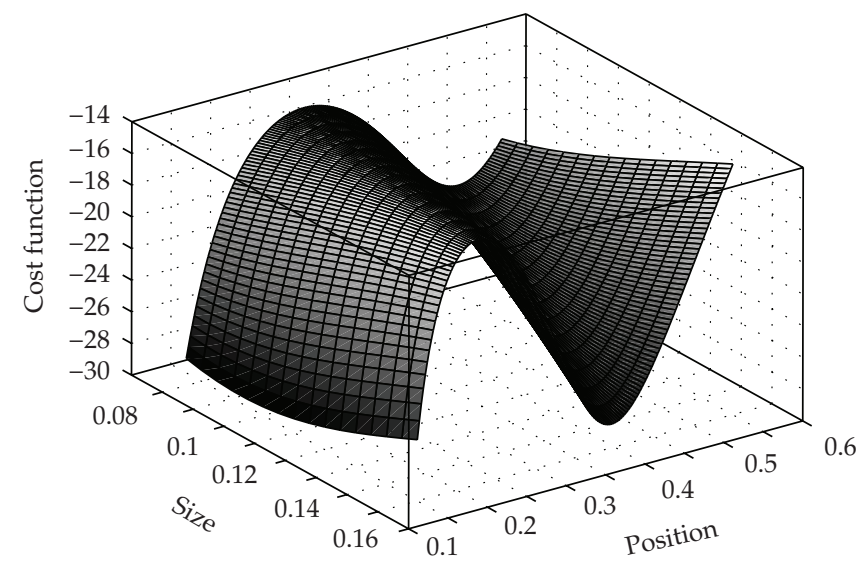

Figure 4: Dissipated energy objective function due to piezoelectric control (case 2).

Table 3: Beam and piezoelectric properties (Example 6.2).

\begin{tabular}{lccc}
\hline & Beam & Actuators & Sensors \\
\hline Young's modulus $(\mathrm{GPa})$ & $65 \times 10^{9}$ & $64 \times 10^{9}$ & $2 \times 10^{9}$ \\
Mass density $\left(\mathrm{kg} \mathrm{m}^{-3}\right)$ & 2890 & 7700 & 1780 \\
Stress constant $d_{31}\left(\mathrm{~m} \mathrm{~V}^{-1}\right)$ & - & $-32 \times 10^{-11}$ & - \\
Strain constant $g_{31}\left(\mathrm{Vm} \mathrm{N}^{-1}\right)$ & - & - & $216 \times 10^{-3}$ \\
Thickness $(\mathrm{m})$ & $1 \times 10^{-3} \rightarrow 0.6 \times 10^{-3}$ & $815 \times 10^{-6}$ & $28 \times 10^{-6}$ \\
Width $(\mathrm{m})$ & $25 \times 10^{-3}$ & $25 \times 10^{-3}$ & $25 \times 10^{-3}$ \\
Length $(\mathrm{m})$ & 0.7 & - & - \\
\hline
\end{tabular}

\section{Manipulators Motion and Control}

\subsection{Equations of Manipulators Motion}

The closed form equations of motion are derived using Lagrange's equations, written as

$$
\frac{d}{d t}\left(\frac{\partial L}{\partial \dot{q}_{i}}\right)-\frac{\partial L}{\partial q_{i}}=F_{i}, \quad i=1,2, \ldots, N+n
$$

where $L=T-U, T$ is the kinetic energy, $U$ the potential energy, $q_{i}$ the generalized coordinate, associated with joint coordinates and link deflections, and $F_{i}$ are the generalized forces. $N$ is the number of links and $n$ the number of modes. It can be written in compact matrix notation [22], resulting

$$
\mathbf{M}_{\mathbf{r}}(\mathbf{q}) \ddot{\mathbf{q}}+\mathrm{C}_{\mathbf{r}}(\mathbf{q}, \dot{\mathbf{q}}) \dot{\mathbf{q}}+K_{\mathrm{r}} \mathbf{q}+\mathrm{D}_{\mathbf{r}} \dot{\mathbf{q}}+\mathbf{g}(\mathbf{q})=\Gamma,
$$

where $\mathbf{q}=[\boldsymbol{\alpha}, \boldsymbol{\eta}]^{\mathrm{T}}$ is the generalized coordinates vector, $\boldsymbol{\alpha} \in \mathfrak{R}^{N}$ is the joint coordinates vector, $\eta \in \Re^{n}$ is the elastic modes coordinates vector, $\mathbf{M}_{\mathbf{r}}(\mathbf{q})$ is the positive definite symmetric inertia matrix, $\mathbf{C}_{\mathbf{r}}(\mathbf{q}, \dot{\mathbf{q}})$ is the Coriolis and centrifugal forces matrix, $\mathbf{g}(\mathbf{q})$ is the gravitational torque vector, $\mathbf{K}_{\mathrm{r}}$ is the positive definite stiffness diagonal matrix, $\mathbf{D}_{\mathbf{r}}$ is the positive semi-definite link diagonal modal damping matrix, and $\Gamma=[\boldsymbol{\tau}, \mathbf{0}]_{(N+n) \times 1}$ is the joint input torque vector. 
Variable tip mass (payload) can be estimated by the change of the fundamental frequency which can be excited by a preliminary perturbation.

\subsection{Tip Position Control}

Under certain conditions, achieving the suppression of elastic link vibrations by the motor torque alone may be very difficult. Hardware limitations, such as motor saturation and motor noise, may prevent the control of high frequency vibration modes. To address these shortcomings we use a hybrid controller consisting of the servo-motor and piezoelectric actuators and sensors bonded to the flexible links as shown in Figure 1. The vibration feedback control voltage to the piezoceramic actuator based on the voltage of the piezofilm sensor is show in Section 3.

The total control pruposes is an uncoupled combination of motor torque control and piezoelectric material control. The various matrices from (7.2), substituting the matrices from (3.3), including the piezoelectric actuator control, can be partitioned as

$$
\begin{array}{cc}
\mathbf{M}_{\mathbf{r}}(\mathbf{q})=\left[\begin{array}{cc}
\mathbf{M}_{\alpha \alpha} & \mathbf{M}_{\alpha \eta} \\
\mathbf{M}_{\alpha \eta}^{\mathrm{T}} & \overline{\mathbf{M}}
\end{array}\right], \quad \mathrm{C}_{\mathbf{r}}(\mathbf{q}, \dot{\mathbf{q}})=\left[\begin{array}{ll}
\mathrm{C}_{\alpha \alpha} & \mathrm{C}_{\alpha \eta} \\
\mathrm{C}_{\eta \alpha} & \mathrm{C}_{\eta \eta}
\end{array}\right], \quad \mathrm{D}_{\mathbf{r}}=\left[\begin{array}{ll}
0 & 0 \\
0 & \overline{\mathrm{D}}
\end{array}\right], \\
\mathbf{K}_{\mathbf{r}}=\left[\begin{array}{cc}
0 & 0 \\
0 & \overline{\mathbf{K}}
\end{array}\right], \quad \mathrm{g}(\mathbf{q})=\left[\begin{array}{l}
\mathrm{g}_{\eta}(\boldsymbol{\alpha}) \\
\mathrm{g}_{\alpha}(\boldsymbol{\eta})
\end{array}\right], \quad \Gamma=\left[\begin{array}{c}
\tau \\
\mathrm{P}^{\mathrm{a}} \mathbf{v}^{\mathrm{a}}(\mathbf{t})
\end{array}\right],
\end{array}
$$

where the indexes $\boldsymbol{\alpha} \boldsymbol{\alpha}, \boldsymbol{\alpha} \boldsymbol{\eta}$ are the terms from the matrices corresponding to rigid body motions and coupling of rigid and flexible motion.

\subsection{Control via State-Dependent Riccati Equations}

The SDRE method is used for positioning control, which implies in a vibration control on the flexible link. In this paper, this method is used to derive the control of the joint torques. The complete control of the tip position includes the joints control by the SDRE method and the piezoelectric actuators. The actuation frequencies ranges of the motor and piezoelectric inserts are chosen to be nonoverlapping, so that their controls are uncoupled.

The dynamic system defined by (7.2) and (7.3) can be parameterized in first-order equations and written in the state-dependent coefficient (SDC) form

$$
\begin{gathered}
\dot{\mathbf{x}}=\mathbf{A}_{\mathbf{r}}(x) \mathbf{x}+\mathbf{B}_{\mathbf{r}}(x) \boldsymbol{\Gamma}, \\
\mathbf{y}=\mathbf{S}(x) \mathbf{x},
\end{gathered}
$$

where $\mathbf{x} \in \mathfrak{R}^{2(N+n)}$ is a state time dependent, $\dot{\mathbf{x}} \in \mathfrak{R}^{2(N+n)}$ is the vector of the first-order time derivatives of the states, $\boldsymbol{\Gamma} \in \boldsymbol{\Theta} \in \mathfrak{R}^{N+m}$ is the control vector, $\boldsymbol{\Theta}$ is the control constraint set. This system represents the constrains from the nonlinear regulator problem, together with $\mathbf{x}\left(t_{0}\right)=\mathbf{x}_{\mathbf{0}}, \quad \mathbf{x}(\infty)=\mathbf{0}$, respectively the initial and final conditions. 
The coefficient dependent matrices are given by

$$
\begin{aligned}
& \mathbf{A}_{\mathbf{r}}(x)=\left[\begin{array}{cc}
\mathbf{0} & \mathbf{I} \\
-\mathbf{M}_{\mathbf{r}}^{-1} \mathbf{K}_{\mathbf{r}} & -\mathbf{M}_{\mathbf{r}}^{-\mathbf{1}}\left(\mathbf{C}_{\mathbf{r}}+\mathbf{D}_{\mathbf{r}}\right)
\end{array}\right], \quad \mathbf{B}_{\mathbf{r}}(x)=\left[\begin{array}{c}
\mathbf{0} \\
\mathbf{M}_{\mathbf{r}}^{-1}
\end{array}\right], \\
& \mathbf{Q}_{\mathbf{r}}(x)=\mathbf{S}^{\mathbf{T}}(x) \mathbf{S}(x), \quad \mathbf{S}(x)=\operatorname{diag}\left\{\sqrt{q_{r k}}\right\}_{k=1, \ldots, 2(N+m)^{\prime}}
\end{aligned}
$$

where $\mathbf{A}_{\mathbf{r}} \in \mathfrak{R}^{2(N+n) \times 2(N+n)}$ and $\mathbf{B}_{\mathbf{r}} \in \mathfrak{R}^{2(N+n) \times 2(m)}$. The state and control dependent coefficients are given by $\mathbf{f}(x)=\mathbf{A}_{\mathbf{r}}(x) \mathbf{x}, \mathbf{b}_{\mathbf{r}}(x)=\mathbf{B}_{\mathbf{r}}(x)$, and $\mathbf{d}(x)=\mathbf{S}(x) \mathbf{x}$ [12]. It is assumed that $\mathbf{f}(\mathbf{0})=\mathbf{0}$, implying that the origin is an equilibrium point.

A state feedback rather that output feedback is adopted to enhance the control performance. The nonquadratic cost function for the regulator problem is given by

$$
J_{r}=\frac{1}{2} \int_{t_{0}}^{\infty}\left[\mathbf{x}^{\mathrm{T}} \mathbf{Q}_{\mathbf{r}}(x) \mathbf{x}+\Gamma^{\mathrm{T}} \mathbf{R}(x) \Gamma\right] d t
$$

where $\mathbf{Q}_{\mathbf{r}}(x) \mathbf{x}$ is semipositive definite matrix and $\mathbf{R}(x)$ positive definite. They are weighting matrices on the outputs and control inputs, respectively. These are the weighting matrices on the output and control input, respectively. They are assumed to be constant for the piecewise linear approach.

Assuming full state feedback, the control law is given by

$$
\Gamma=-\mathbf{R}^{-1}(x) \mathbf{B}_{\mathbf{r}}^{\mathrm{T}}(x) \Pi(x) \mathbf{x}
$$

The state-dependent Riccati equation to obtain $\Pi(x)$ is given by

$$
\mathbf{A}_{\mathbf{r}}^{\mathrm{T}}(x) \Pi(x)+\Pi(x) \mathbf{A}_{\mathbf{r}}(x)-\Pi(x) \mathbf{B}_{\mathbf{r}}(x) \mathbf{R}^{-1}(x) \mathbf{B}_{\mathbf{r}}^{\mathrm{T}}(x) \Pi(x)+\mathbf{Q}_{\mathbf{r}}(x)=0 .
$$

We remark four theorems $[13,14]$ about SDRE technique.

Theorem 7.1. In the neighborhood $\Sigma$ about the origin the SDRE method guarantees a closed-loop solution, local asymptotic stability.

Note that the closed-loop solution is given by

$$
\dot{\mathbf{x}}=\mathbf{A}_{\mathbf{r}}(x) \mathbf{x}-\mathbf{B}_{\mathbf{r}}(x)-\mathbf{R}^{-1}(x) \mathbf{B}_{\mathbf{r}}^{\mathrm{T}}(x) \Pi(x) \mathbf{x}=\mathbf{A}_{\mathbf{d}}(x) \mathbf{x} .
$$

$\mathbf{A}_{\mathbf{d}}(x)$ is guaranteed to be stable at every point $x$ from Riccati equation Theory. And in a neighborhood about the origin it is shown in $[13,14]$ that the system $(7.9)$ is asymptotic stabile.

Theorem 7.2. In the scalar case, the SDRE method reaches the optimal solution of the feedback regulator problem performance index (7.5), even when $\mathbf{Q}_{\mathbf{r}}$ and $\mathbf{R}$ are functions of $x$.

There exists only one SDC parameterization in the scalar case $a_{r}(x)=f(x) / x$. It can be show that the state-dependent Riccati equation from this parameterization has a positive-definite solution. The first and the second necessary conditions for optimality are satisfied. This theorem is proved in [13]. 
Theorem 7.3. In general multivariable case, the SDRE nonlinear feedback controller satisfies the first necessary condition for optimality, $\partial \mathbf{H} / \partial \Gamma=\mathbf{0} \mathbf{H}$ is the Hamiltonian from the problem (7.4)-(7.6), while the second necessary condition for optimality, $\boldsymbol{i}=-\partial \mathbf{H} / \partial x$, is asymptotically satisfied at a quadratic rate as $\mathbf{x}$ goes to zero.

The Hamiltonian is given by

$$
\mathbf{H}(x, \Gamma, \lambda)=\frac{1}{2}\left(\mathbf{x}^{\mathrm{T}} \mathbf{Q}_{r}(x) \mathbf{x}+\Gamma^{\mathrm{T}} \mathbf{R}(x) \Gamma\right)+\mathcal{\lambda}^{T}\left(\mathbf{A}_{\mathbf{r}}(x) \mathbf{x}+\mathbf{B}_{\mathbf{r}}(x) \Gamma\right),
$$

which implies that

$$
\begin{gathered}
\mathbf{H}_{\Gamma}=\mathbf{R}(x) \boldsymbol{\Gamma}+\mathbf{B}_{\mathbf{r}}(x) \mathcal{\Lambda}=0, \\
\Gamma=-\mathbf{R}^{-1}(x) \mathbf{B}_{\mathbf{r}}^{\mathrm{T}}(x) \boldsymbol{\lambda} .
\end{gathered}
$$

Then one assume the costate

$$
\lambda=\Pi(x) \mathbf{x}
$$

Substituting $\boldsymbol{\lambda}$ into $\Gamma$ yields the SDRE controller.

Theorem 7.4. The system (7.4) is pointwise controllable and observable, for a region in neighborhood $\sum$ about the origin.

For controllability this mean full rank for the matrix $\left[\mathbf{B}_{\mathbf{r}} \vdots \mathbf{A}_{\mathbf{r}}^{l} \mathbf{B}_{\mathbf{r}}\right]_{l=1, \ldots, 2(N+n-1)}$ from the static problem $\dot{\mathbf{x}}=\mathbf{A}_{\mathbf{r}} \mathbf{x}+\mathbf{B}_{\mathbf{r}} \Gamma$, in this neighborhood. SDRE method considers a solution for this static pointwise problem, for small time interval.

The detailed proofs from these theorems can be seen in $[13,14]$.

In fact, in our case we cannot obtain the Riccati solution analytically, due to its complex invertibility of the inertia matrix, therefore we used some numerical features, described in the following steps. The SDRE technique to obtain a suboptimal solution for the flexible manipulator problem with piezoelectric actuators has the following procedure [12].

Step 1. Define the space-state model of the manipulator in the state-dependent coefficient form (7.4).

Step 2. Measure the state of the system $x(t)$, that is, define $\mathbf{x}(\mathbf{0})=\mathbf{x}_{\mathbf{0}}$, and choose the coefficients of weight matrices $\mathbf{Q}_{\mathrm{r}}$ and $\mathbf{R}$.

Step 3. Solve the Riccati equation (7.8) for the state $\mathbf{x}(t)$ considering pointwise static solutions, that is, solve $A_{r}^{T} \Pi+\Pi A_{r}-\Pi B_{r} R^{-1} B_{r}^{T} \Pi+Q_{r}=0$ for each step.

Step 4. Calculate the input signal from (7.7).

Step 5. Integrated the system (7.4) and update the state of the system $\mathbf{x}(t)$ with these results. Go to Step 3. 


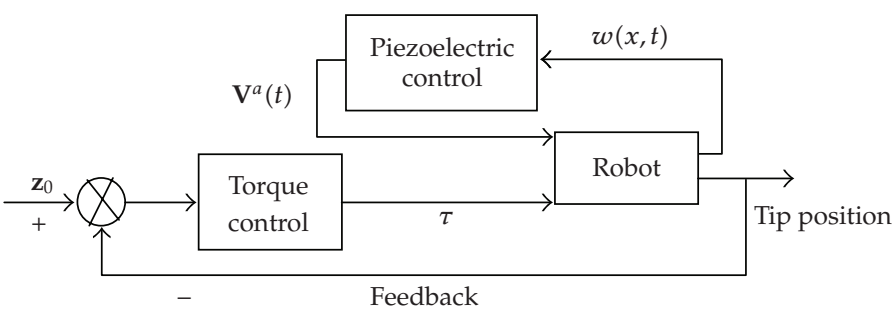

Figure 5: Block-diagram of the proposed control algorithm.

\section{Simulations and Results of the Flexible Manipulator Control}

The optimization and control laws were tested on a single flexible link and on a simplified planar robot model with a rigid first link and a flexible second link. Gravitational effects were ignored; the motion of the flexible beam is in the horizontal plane. The generalized coordinate vector is $\mathbf{q}=\left(\alpha_{1}, \alpha_{2}, \eta_{1}, \eta_{2}\right)$. The inertial matrix, the Coriolis, and centrifugal effects matrix are taken from [23]. Dimensional and mechanical properties of the aluminium flexible link and piezoelectric materials are shown in Table 3.

The proposed control take an initial position $\mathbf{z}_{0}$ for the two link manipulator and drives the tip to a final desired position, without any trajectory constraint, which means that the control tries to find the minimum energy trajectory. Then the optimal problem, for pointwise static solutions, can be reformulated as minimizing the cost functional

$$
J_{z}=\frac{1}{2} \int_{0}^{\infty}\left[\mathbf{z}^{\mathrm{T}} \mathbf{Q}_{\mathbf{r}} \mathbf{z}+\Gamma^{\mathrm{T}} \mathbf{R} \Gamma\right] d t
$$

such that $\dot{\mathbf{z}}=\mathbf{A}_{\mathrm{r}} \mathbf{z}+\mathbf{B}_{\mathbf{r}} \boldsymbol{\Gamma}, \mathbf{z}(\mathbf{0})=\mathbf{z}_{\mathbf{0}}, \mathbf{z}(\infty)=\mathbf{0}$, where the vector $\mathbf{z}=\left[\mathbf{x}-\mathbf{x}_{\mathbf{d}}\right], \mathbf{x}_{\mathbf{d}}$ is the desired tip position with joint coordinates and zero for the modal deflections. Assuming the initial conditions, the next state $\mathbf{z}(t)$ for each step is obtained considering the control by the torques of the joints and the feedback of the piezoelectric material, as shown in Figure 5.

The resulting equation was coded into a Matlab software, where the fourth-order Runge-Kutta method was used to integrate by solving the equations. The Riccati equation was solved using the Matlab function "LQR."

The lower fundamental modes are responsible for most of the link's tip displacement, therefore the first two eigenfunctions are considered in the paper.

\subsection{Simulations of the Manipulator Controlled Dynamics}

The first simulation (Figure 6), shows the dynamic behaviour, of the flexible link, without vibration control.

The second simulation (Figures $7(\mathrm{a})-7(\mathrm{~d})$ ), shows the displacement at joints when the torque control is used. It is simulated for two different cases. At first moment, (Figures 7(a) and $7(b)$ ), the initial joint coordinates are $\alpha_{1}=5 \pi / 4, \alpha_{2}=-\pi$ and deflections $\eta_{1}=0, \eta_{2}=0$. In this position the links are overlapping by $\pi / 4$ at the axes $x$ and $y$. The desired point, for the joints, to drive the robot arm is $\alpha_{1}=\pi / 4, \alpha_{2}=0$, and for the deflections $\eta_{1}=0, \eta_{2}=0$. At the second moment, (Figures (c) and (d)), the initial point is $\alpha_{1}=0, \alpha_{2}=0$, and the desired point $\alpha_{1}=1, \alpha_{2}=1$. The robot arm positions for these two cases are shown in (Figures 7(e)-7(h)). 


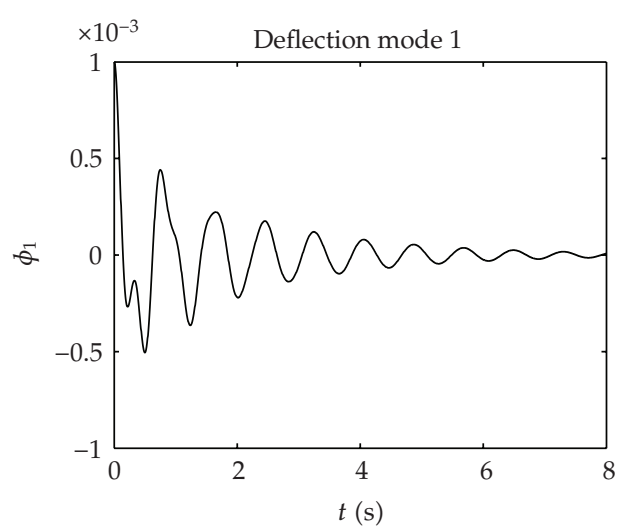

(a)

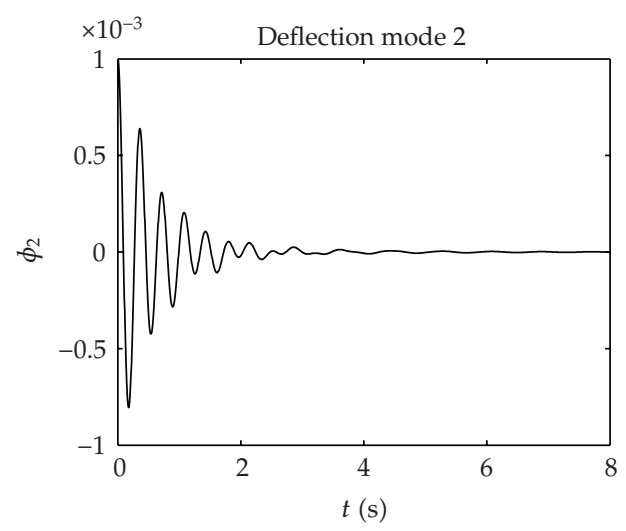

(b)

Figure 6: Deflection of $\phi_{1}$ and $\phi_{2}$.

The matrices $\mathbf{Q}_{\mathbf{r}}$ and $\mathbf{R}$, for the pointwise static problem, after some trial the matrices, were chosen as $\mathbf{Q}=\operatorname{diag}\{50, \ldots, 50\}$ and $\mathbf{R}=\operatorname{diag}\{500, \ldots, 500\}$.

The third simulation, (Figures 8 and 9), show the vibration control of the flexible link with the torque feedback gain. The initial conditions are the same as in the first case above. In space coordinates the initial point is $x_{0}=y_{0}=0.2828$, and the desired point $x_{d}=y_{d}=0.7071$.

Figure 9 shows that the endpoint of the robot initially vibrates, but it is controlled in a short time.

The next simulation, (Figure 10), shows the vibration control of the flexible link with the torque feedback gain and including the piezoelectric constant amplitude controller. The initial conditions are the same as in Figures $7(\mathrm{a})$ and $7(\mathrm{~b})$. The gain $\mathrm{G}=\operatorname{diag}\{0.1,0.1\}$.

An important aspect, which was not modeled in this work, is the fact that the strain of the piezoelectric actuator is limited; however the chosen feedback gains do not extrapolate these limitations.

The actuator feedback gain $\mathbf{V}^{\mathbf{a}}(t)$ from the control system with piezoelectric control is shown in Figure 11. It is noticeable that the force applied of the actuator produces a moment that opposes the link deflection.

In a flexible link manipulator, system parameters, like the payload, cannot be known exactly, and this can introduce significant uncertainties in the dynamic model. Also, it can be the deviations from nominal values of the material properties or physical parameters; these can affect the efficient of the simulation when compared with a practical case.

We can note that the state feedback is given by the analytical simulation of the physical system. It could be interesting to introduce also a control provided from the measured response of the system, through the piezofilm, but we do not consider this in the paper.

Using the SDRE method implies that the controllability of the static problem depends on the size from the time step. In this paper this chosen size was $0.001 \mathrm{sec}$. Controllability is lost for large time steps. In our case, with high frequencies, the time step is also important for characterizing correct frequency period.

The choice of the best values for the state weighting matrix $Q_{r}$ is very important. A good choice can improve the efficiency of the controllers. In this paper we have tested some weighting matrices and concluded that, for our control design, the good results are obtained with values around $\mathbf{Q}_{\mathbf{r}}=\operatorname{diag}\{50, \ldots, 50\}$. Smaller or greater values affect the control efficiency. 


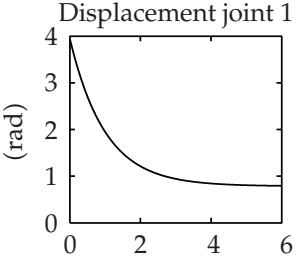

(s)

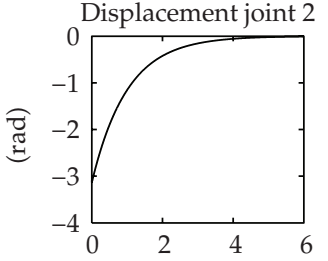

(s)

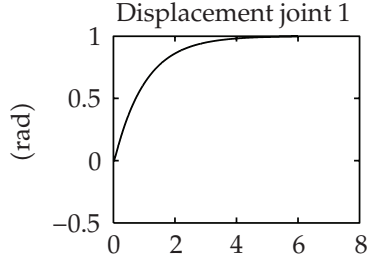

(s)

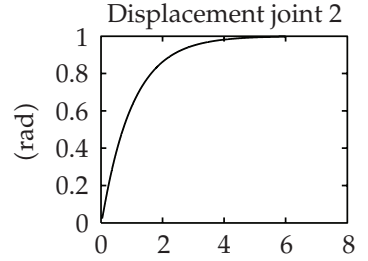

(s)

(a)

(b)

(c)

(d)

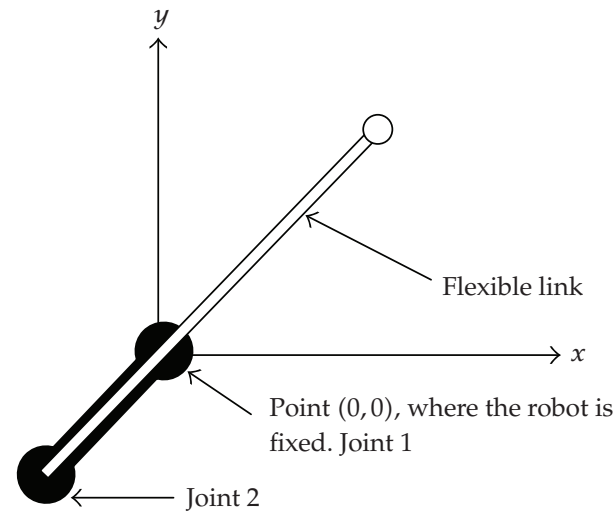

(e) Initial position for the first case

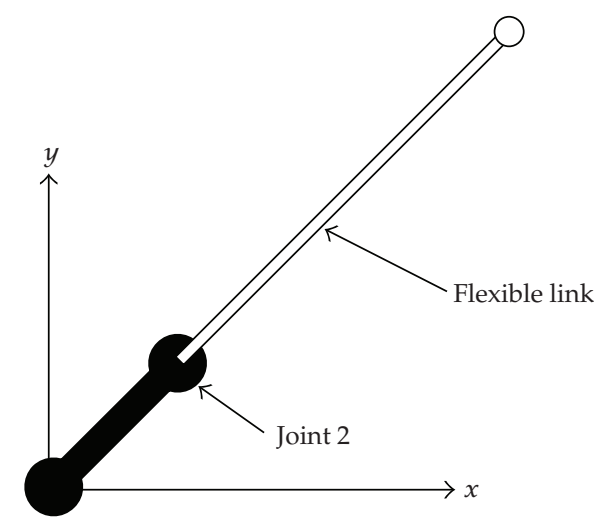

(f) Final position for the first case

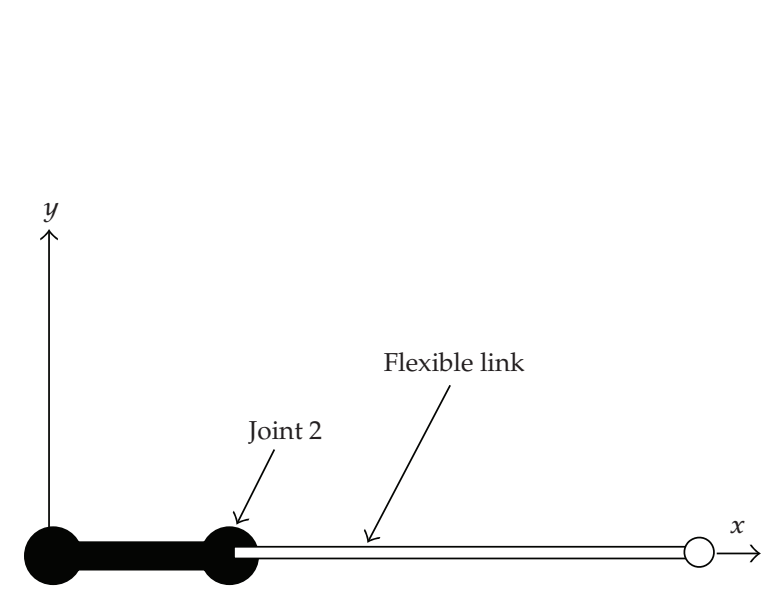

(g) Initial position for the second case

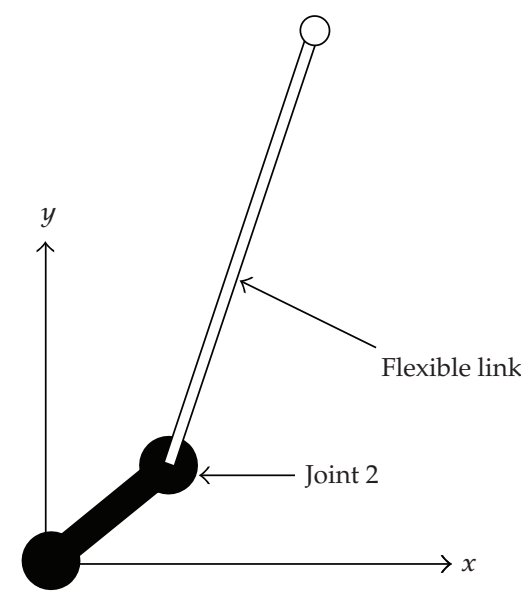

(h) Final position for the second case

Figure 7: Joint displacement.

The stability analysis for this system may be examined around the origin [12]. The linearization technique was used for

$$
\begin{aligned}
\mathbf{f}(x) & =\mathbf{A}_{\mathbf{r}}(x) \mathbf{x}, \quad \mathbf{w}_{\mathbf{r}}(x, \Gamma)=\mathbf{B}_{\mathbf{r}}(x) \Gamma, \\
J_{f} & =\left[\frac{\partial \mathbf{f}}{\partial \mathbf{x}}\right]_{\mathbf{x}=\mathbf{0}}, \quad J_{h}=\left[\frac{\partial \mathbf{w}_{\mathbf{r}}}{\partial \Gamma}\right]_{\mathbf{x}=\mathbf{0}},
\end{aligned}
$$




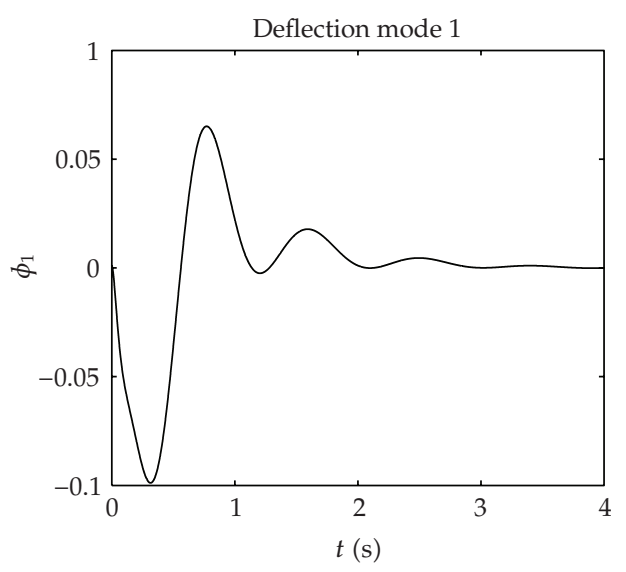

(a)

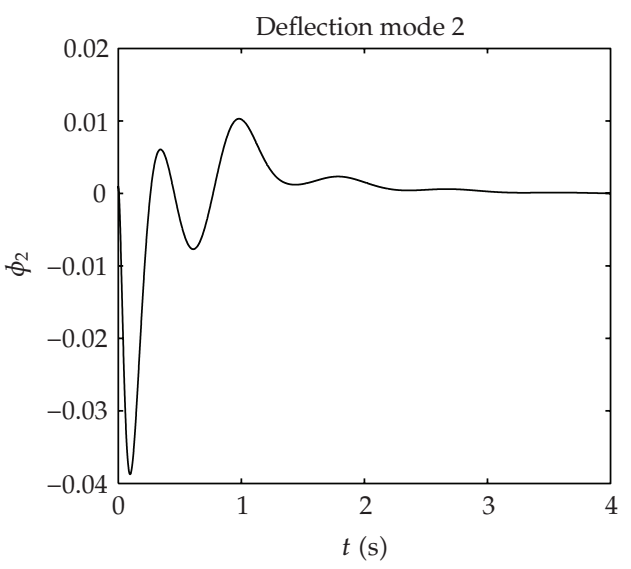

(b)

Figure 8: Step response of the flexible link using SDRE.

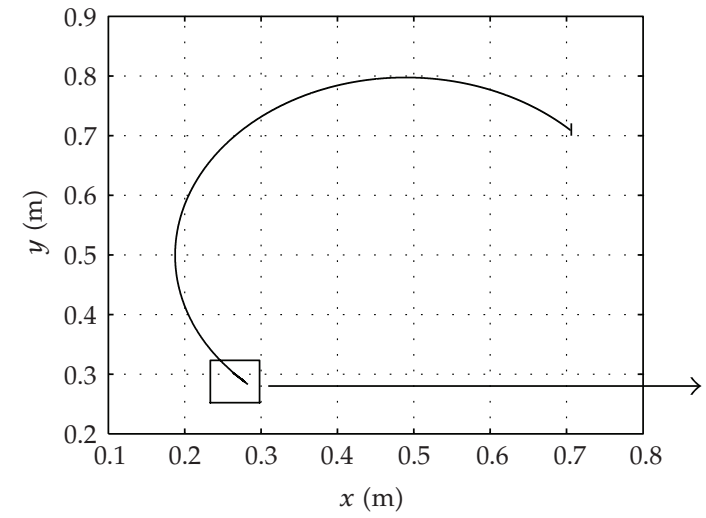

(a)

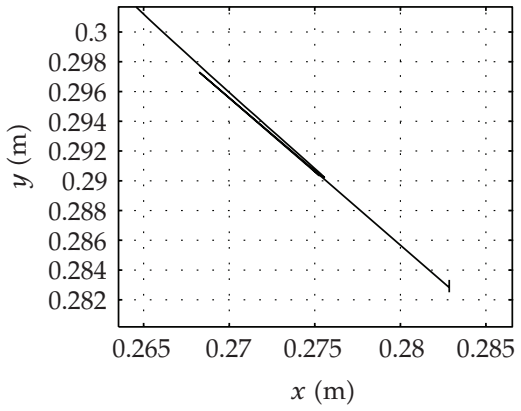

(b)

Figure 9: Trajectory in space coordinates an endpoint of the robot arm, in meters. The start point and desired point are set by rectangles. The inset shows the start of the movement in a larger scale for better visualization.

where $J_{f}$ and $J_{h}$ are the Jacobian matrices of $\mathbf{f}(x)$ and $\mathbf{w}_{\mathbf{r}}(x, \Gamma)$ at $\mathbf{x}=\mathbf{0}$, respectively. If the eigenvalues of the Jacobian have negative real parts, the point $\mathbf{x}=\mathbf{0}$ is a locally stable equilibrium point. If one of the real parts are positive, then the point $\mathbf{x}=\mathbf{0}$ is an unstable equilibrium point. In our case, $J_{f}=\mathbf{A}_{\mathbf{r}}(\mathbf{0}), J_{h}=\mathbf{B}_{\mathbf{r}}(\mathbf{0})$. Then, a necessary condition for a local stability is that the pair $\left\{\mathbf{A}_{\mathbf{r}}(\mathbf{0}), \mathbf{B}_{\mathbf{r}}(\mathbf{0})\right\}$ has to be stabilizable. It was obtained one positive eigenvalue, so that we have an unstable equilibrium point at the origin. Even so, the linearized system is pointwise controllable and observable for a region of interest $\Sigma$. This fact is shown in [12], and we have also verified the controllability for our system. The stability is obtained by full state feedback gain $\Gamma=-\mathbf{R}^{-1}(x) \mathbf{B}_{\mathbf{r}}^{\mathrm{T}}(x) \Pi(x) \mathbf{x}$. 


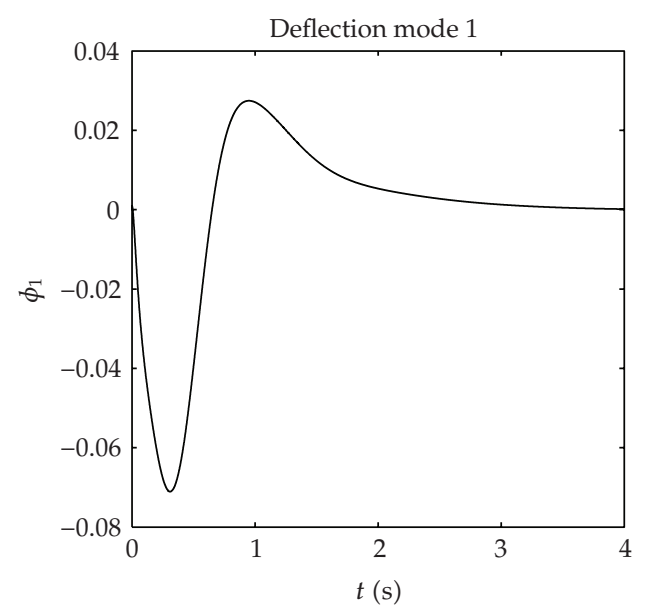

(a)

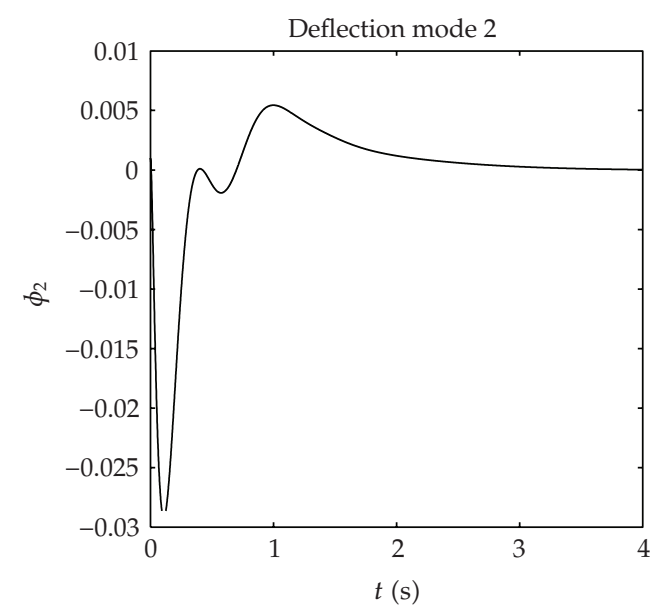

(b)

Figure 10: Step response of the flexible link using SDRE and piezoelectric controllers.

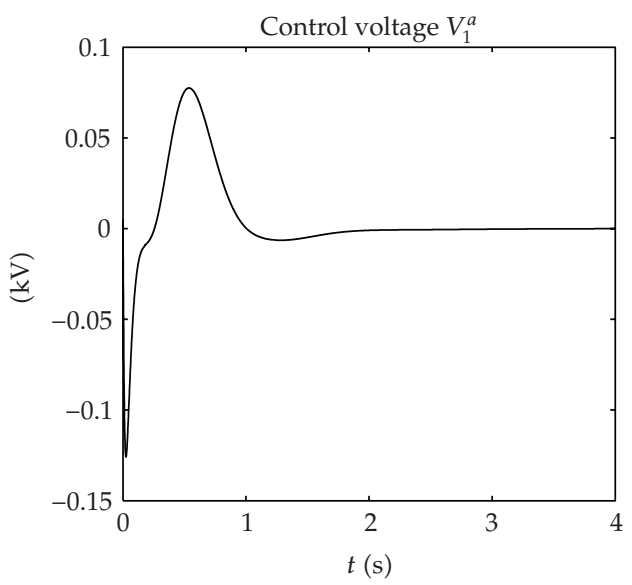

(a)

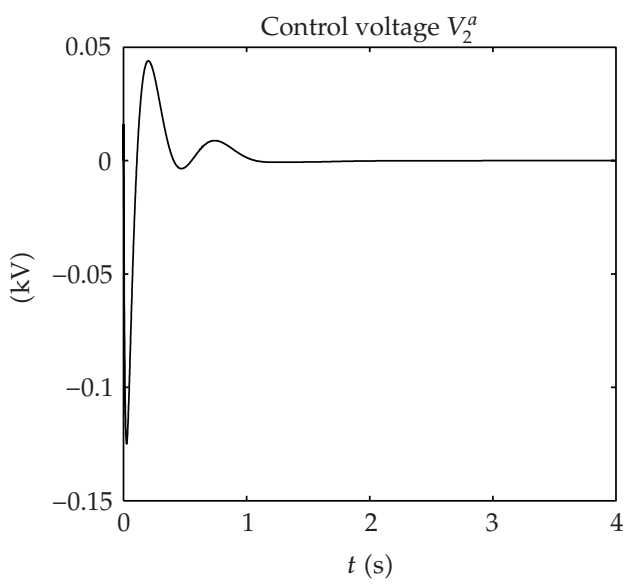

(b)

Figure 11: Actuator feedback control voltage response for the beam in vibration.

\section{Conclusions}

This paper introduces a technique for optimization of placement and size of piezoelectric material for the optimal vibration control of flexible robot links. A GA technique was used on the optimal control strategies for choosing the best location and size for some given discretization. Piezoelectric actuators and sensors are added to the system to control the frequency vibrations considering that the properties of the structure changes where the actuators and sensors are added. This technique can be used to build lightweight structures with controlled vibration levels, as manipulators with flexible links, while preserving the stiffness and precision. It also reduces the energy consumption and suits the needs for aerospace systems or for tasks that demand lightness, precision, and agility. 
The simulations for the control system have confirmed effectiveness for this control technique. The numerical results indicate that the location and size of the sensor/actuators may have significant influence on the integrated system control performance. Also the feedback gain affects directly the control efficiency. The suboptimal State-Dependent Riccati Equation technique, together with the piezoelectric actuators and sensors, can control the vibrations on the flexible link in a short time span.

The control with the motor torques alone could be efficient for low frequencies, but for high frequencies (mainly) the simulations show that piezoelectric actuators increase the control effectivity. The control using just motor torques can be more complicated in some practical applications, where the frequent torque change requires more robust motors. Piezoelectric actuators are more efficient in high frequencies.

The results in the paper show that the SDRE method is at least as efficient with vibration control as other robust control methods, although it requires some heuristic weighting matrices.

\section{Acknowledgments}

The authors A. Molter, acknowledge, the financial support of FAPERGS (Fundação de Amparo à Pesquisa do Estado do Rio Grande do Sul)- Porto Alegre- Rio Grande do SulBrazil, project: 10/0091-3, the Karl Franzens Universität Graz-Austria, and the START project: Interfaces and Free Boundaries. The authors would like to acknowledge the valuable discussions with M. Hintermüller (Graz-Austria).

\section{References}

[1] Y. Li, J. Onoda, and K. Minesugi, "Simultaneous optimization of piezoelectric actuator placement and feedback for vibration suppression," Acta Astronautica, vol. 50, no. 6, pp. 335-341, 2002.

[2] D. Sun, J. K. Mills, J. Shan, and S. K. Tso, "A PZT actuator control of a single-link flexible manipulator based on linear velocity feedback and actuator placement," Mechatronics, vol. 14, no. 4, pp. 381-401, 2004.

[3] S. X. Xu and T. S. Koko, "Finite element analysis and design of actively controlled piezoelectric smart structures," Finite Elements in Analysis and Design, vol. 40, no. 3, pp. 241-262, 2004.

[4] G. L. C. M. Abreu, J. F. Ribeiro, and V. Steffen, "Experiments on optimal vibration control of a flexible beam containing piezoelectric sensors and actuators," Shock and Vibration, vol. 10, no. 5-6, pp. 283-300, 2003.

[5] Q. Hu and G. Ma, "Variable structure maneuvering control and vibration suppression for flexible spacecraft subject to input nonlinearities," Smart Materials and Structures, vol. 15, no. 6, pp. 1899-1911, 2006.

[6] K. Ramesh Kumar and S. Narayanan, "Active vibration control of beams with optimal placement of piezoelectric sensor/actuator pairs," Smart Materials and Structures, vol. 17, no. 5, Article ID 055008, 15 pages, 2008.

[7] Y. Yang, Z. Jin, and C. K. Soh, "Integrated optimal design of vibration control system for smart beams using genetic algorithms," Journal of Sound and Vibration, vol. 282, no. 3-5, pp. 1293-1307, 2005.

[8] J.-J. Wei, Z.-C. Qiu, J.-D. Han, and Y.-C. Wang, “Experimental comparison research on active vibration control for flexible piezoelectric manipulator using fuzzy controller," Journal of Intelligent and Robotic Systems, vol. 59, no. 1, pp. 31-56, 2010.

[9] S. B. Choi, S. S. Cho, H. C. Shin, and H. K. Kim, “Quantitative feedback theory control of a single-link flexible manipulator featuring piezoelectric actuator and sensor," Smart Materials and Structures, vol. 8, no. 3, pp. 338-349, 1999. 
[10] V. Bottega, A. Molter, J. S. O. Fonseca, and R. Pergher, "Vibration control of manipulators with flexible nonprismatic links using piezoelectric actuators and sensors," Mathematical Problems in Engineering, vol. 2009, Article ID 727385, 16 pages, 2009.

[11] H. K. Kim, S. B. Choi, and B. S. Thompson, "Compliant control of a two-link flexible manipulator featuring piezoelectric actuators," Mechanism and Machine Theory, vol. 36, no. 3, pp. 411-424, 2001.

[12] A. M. Shawky, A. W. Ordys, L. Petropoulakis, and M. J. Grimble, "Position control of flexible manipulator using non-linear $H_{\infty}$ with state-dependent Riccati equation," Proceedings of the Institution of Mechanical Engineers. Part I: Journal of Systems and Control Engineering, vol. 221, no. 3, pp. 475-486, 2007.

[13] C. P. Mracek and J. R. Cloutier, "Control designs for the nonlinear benchmark problem via the statedependent Riccati equation method," International Journal of Robust and Nonlinear Control, vol. 8, no. 4-5, pp. 401-433, 1998.

[14] H. T. Banks, B. M. Lewis, and H. T. Tran, "Nonlinear feedback controllers and compensators: a statedependent Riccati equation approach," Computational Optimization and Applications, vol. 37, no. 2, pp. 177-218, 2007.

[15] C. L. Dym and I. H. Shames, Solid Mechanics. A Variational Approach, McGraw-Hill, New York, NY, USA, 1973.

[16] E. F. Crawley and J. de Luis, "Use of piezoelectric actuators as elements of intelligent structures," AIAA Journal, vol. 25, no. 10, pp. 1373-1385, 1987.

[17] H. T. Banks, R. C. Smith, and Y. Wang, Smart Material Structures: Modeling, Estimation and Control, John Wiley \& Sons, Paris, France, 1996.

[18] K. K. Denoyer and M. K. Kwak, “Dynamic modelling and vibration suppression of a slewing structure utilizing piezoelectric sensors and actuators," Journal of Sound and Vibration, vol. 189, no. 1, pp. 13-31, 1996.

[19] N. Dakev, "Modeling and optimization of dissipative properties of industrial articulated manipulators," in Vibrations and Dynamics of Robotic and Multibody Structures, vol. 57, pp. 7-14, ASME, New York, NY, USA, 1993.

[20] F. Fahroo and M. A. Demetriou, "Optimal actuator/sensor location for active noise regulator and tracking control problems," Journal of Computational and Applied Mathematics, vol. 114, no. 1, pp. 137$158,2000$.

[21] K. Deb, "An efficient constraint handling method for genetic algorithms," Computer Methods in Applied Mechanics and Engineering, vol. 186, no. 2-4, pp. 311-338, 2000.

[22] D. Halim and S. O. Reza Moheimani, "An optimization approach to optimal placement of collocated piezoelectric actuators and sensors on a thin plate," Mechatronics, vol. 13, no. 1, pp. 27-47, 2003.

[23] V. Bottega, R. Pergher, and J. S. O. Fonseca, "Simultaneous control and piezoelectric insert optimization for manipulators with flexible link," Journal of the Brazilian Society of Mechanical Sciences and Engineering, vol. 31, no. 2, pp. 105-116, 2009. 


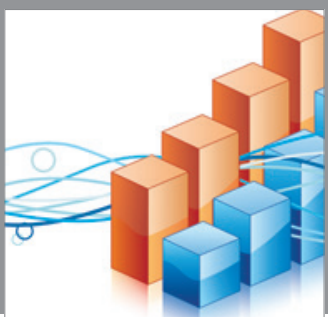

Advances in

Operations Research

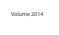

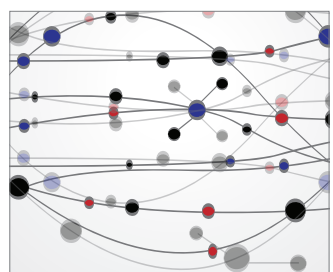

\section{The Scientific} World Journal
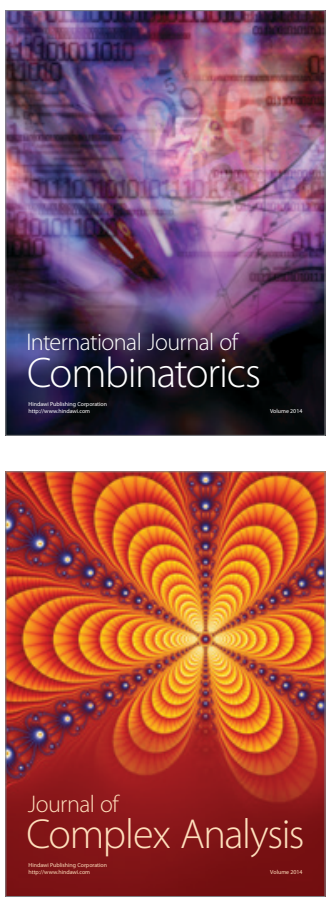

International Journal of

Mathematics and

Mathematical

Sciences
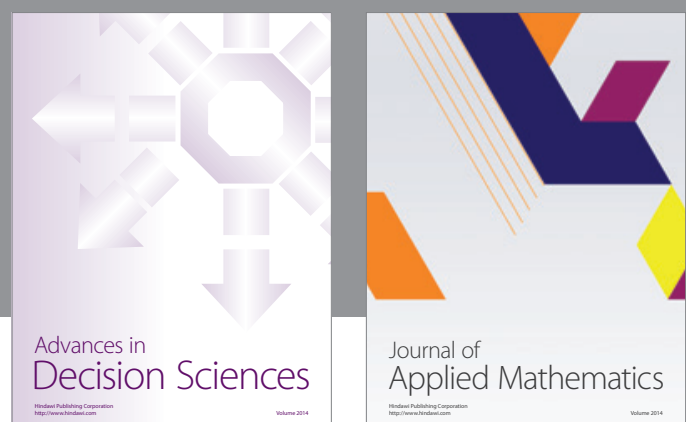

Journal of

Applied Mathematics
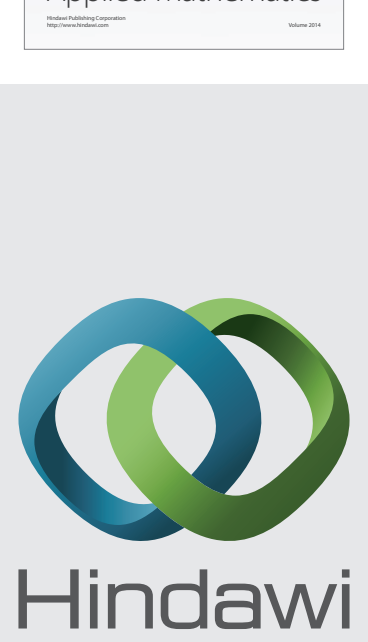

Submit your manuscripts at http://www.hindawi.com
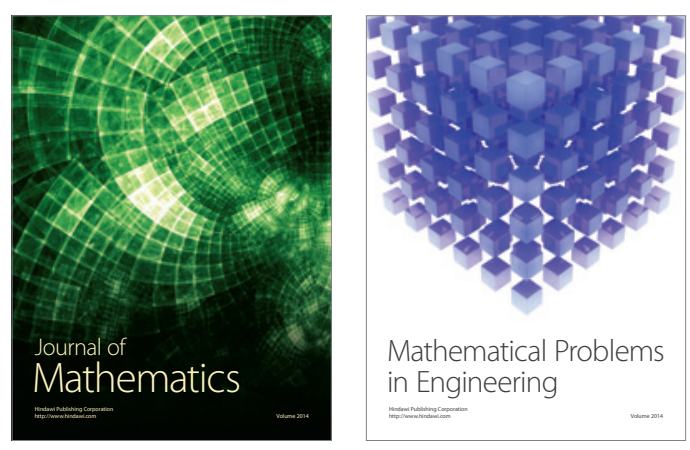

Mathematical Problems in Engineering
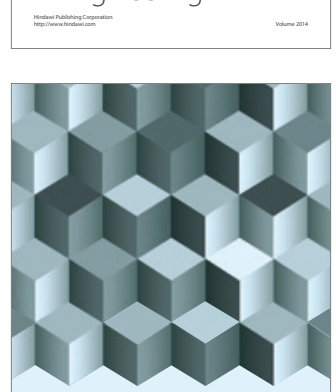

Journal of

Function Spaces
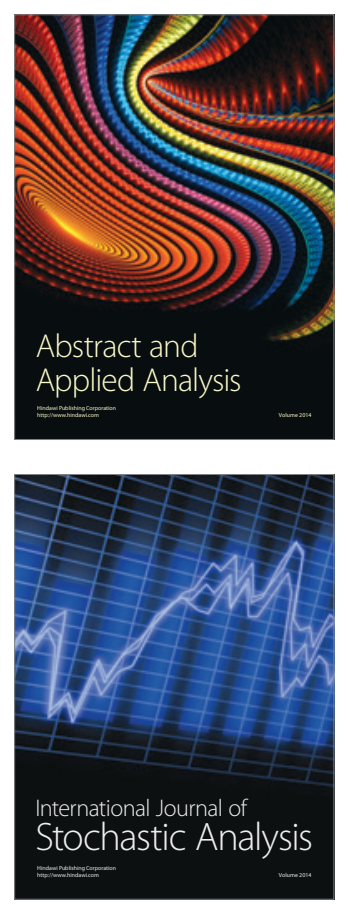

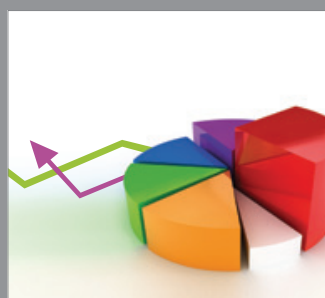

ournal of

Probability and Statistics

Promensencen
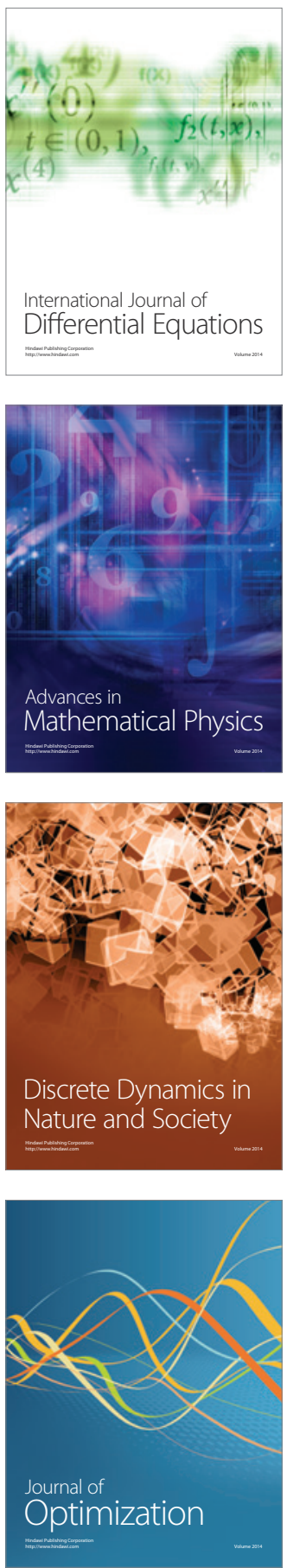